\title{
Browning the Epicardial Adipose Tissues in Cardiovascular Regulation
}

\author{
Hui-Chen Ku and Ching-Feng Cheng* \\ Department of Pediatrics, Taipei Tzu Chi Hospital, Taipei, Taiwan
}

Received: 制: December 18, 2018; Published: 制: January 02, 2019

*Corresponding author: Ching-Feng Cheng, Department of Pediatrics, Taipei Tzu Chi Hospital, Buddhist Tzu Chi Medical Foundation, 289, Jianguo Road, Xindian District, New Taipei City, 23142, Taiwan

\begin{abstract}
Epicardial adipose tissue (EAT) is located between the myocardium and the visceral layer of pericardium. EAT is characterized by increased rate of fatty acid metabolism and increased expression of thermogenic genes. EAT and the myocardium share the same microcirculation, suggesting a close interaction between them. Under normal physiological conditions, EAT produces anti-inflammatory or anti-atherosclerotic cytokines to exert its cardioprotective effects. Although many clinical studies have found significant associations between increased EAT mass and coronary artery disease (CAD). There is no direct evidence that inflammatory EAT worsens CAD. This short review focuses on the emerging physiological and pathophysiological role of EAT and potential pre-clinical application of EAT re-browning for treatment of cardiovascular disease.
\end{abstract}

Keywords: Epicardial Adipose Tissue; Browning; Cardiovascular Disease

Abbreviations: EAT: Epicardial Adipose Tissue; CAD: Coronary Artery Disease; BAT: Brown Adipose Tissue; UCP1: Uncoupling Protein 1; PRDM16: PR Domain Containing 16; WAT: White Adipose Tissue; TAG: Triacylglycerol; FFA: Free Fatty Acid; SAT: Subcutaneous Adipose Tissue; ROS: Reactive Oxygen Species

\section{Introduction}

\section{Epicardial Adipose Tissue (EAT) and Cardiac Function}

Epicardial adipose tissue (EAT) is the fat depot located between the myocardium and the visceral layer of the pericardium and generally exists in the atrioventricular and interventricular grooves [1,2]. The role of EAT within the heart is complicated, but can be commonly distinguished by mechanical, thermogenic, metabolic, and endocrine/paracrine functions [3,4]. EAT functions mechanically to protect the coronary artery against the torsion induced by the arterial pulse wave and myocardial contraction [5]. In addition, the studies from Sacks et al. had suggested that EAT functions similarly to brown adipose tissue (BAT) and yield heat directly to the myocardium conferring a survival benefit by protecting the heart from ischemia or hypoxia [6]. Higher expression levels of BAT-specific genes, such as uncoupling protein 1 (UCP1), PR domain containing 16 (PRDM16) and PPAR- $\gamma$ coactivator- $1 \alpha$ (PGC-1 $\alpha$ ) were observed in human EAT than in other fat depots [6]. Whether EAT is a BAT or functions as a BAT-like fat depot needs further investigation [7].

Metabolically, EAT exhibits high rates of white adipose tissue (WAT) lipogenesis and lipolysis, acting as local triacylglycerol (TAG) store and as a buffer against toxic levels of FFA, in both myocardium and arteries [8]. EAT is abundant in saturated fatty acids and has the highest rate of free fatty acid (FFA) release and uptake of all fat depots $[9,10]$. It is widely known that the energy production in the heart is primarily generated by FFA oxidation, especially during the period of high demand [4,9]. EAT can produce several adipokines and inflammatory cytokines which generate potential interactions through apocrine or paracrine effect between EAT and myocardium $[11,12]$. Under normal physiological conditions, EAT produces anti-inflammatory or anti-atherosclerotic cytokines, such as adiponectin and adrenomedullin, to exert its cardioprotective effects $[13,14]$. Moreover, McAninch et al. had shown that EAT is a highly inflammatory tissue enriched with genes involved in coagulation, endothelial function, immune signaling and apoptosis compared to subcutaneous adipose tissue (SAT) [15].

\section{EAT in the Pathogenesis of Heart Diseases}

The increment of EAT causes additional mass on both ventricles that can enhance the cardiac work demands and left ventricular hypertrophy [16]. EAT thickness is positively correlated with myocardial fat and may regulate cardiomyocyte function [17]. EAT can release FFA into the bloodstream, perturbing vascular homeostasis and endothelial dysfunction, and causing 
coronary artery disease (CAD) and hypoxia [18,19]. Increased lipid accumulation into cardiomyocytes may lead to cardiac steatosis. Several animal studies had shown that the harmful consequences of lipid overload accompanying obesity, such as diabetes and insulin resistance, that can cause cardiomyocyte apoptosis, cardiac fibrosis and impaired cardiac contractile function [20,21]. Hirata et al. had found that patients with advanced CAD have more inflammatory M1 macrophages but fewer anti-inflammatory M2 macrophages in EAT compared to subjects without CAD [22]. The transition of macrophages from M2 to M1 indicated the macrophage-regulated inflammation in epicardial fat. EAT in CAD patients showed increased expression of genes involved in oxidative stress and increased levels of reactive oxygen species (ROS) products than SAT [23]. Since higher oxidative stress could activate inflammatory signals in EAT and lead to the development and progression of CAD.

\section{Browning EAT to Reduce cardiovascular Risk}

A variety of studies indicate that increasing brown and/or beige adipose mass and activity is a practical target to ameliorate obesity and related cardiac disease [24,25]. The beneficial role of BAT from animal studies showed that its activation reduces hypercholesterolemia and prevents the development of atherosclerosis [26]. Dozio et al. had shown that EAT of patients with CAD is associated with a brown-to-white trans-differentiation characterized by decreased expression levels of thermogenic genes and upregulation of white adipogenesis [27]. This brown-to-white phenotypic transition is associated with a significant increase in ROS production in EAT [27]. Furthermore, transplantation of BAT improves body metabolism and the function of the heart and other WAT fat depots [28]. Therefore, it could be hypothesized that rebrowning of EAT in obesity and CAD individuals may improve the hypoxic, inflammatory microenvironment disturbing the vasculature and causing coronary atherosclerosis.

\section{Conclusion}

The pharmaceutical treatment of EAT in cardiovascular diseases is still unclear. Although the strong clinical correlations exit, there is no direct proof that inflammatory EAT worsens CAD. Since mice have a limited amount of EAT, most of the studies on EAT have been performed in humans. The causative role of EAT in CAD has not been investigated. Therefore, the use of geneticallyengineered animal models, such as animals which develop atherosclerosis, diabetes or hypercholesterolemia, would enlarge our understanding of the role of EAT in the cardio-metabolic diseases. The re-browning of EAT may be obtained using a variety of dietary, environmental and pharmacological approaches. Future pre-clinical trials should be designed to examine the effects of the proper interventions on the EAT prior to cardiac surgeries and establish the modern intervention to facilitate the browning of EAT for reducing the cardiovascular risk.

\section{References}

1. Iacobellis G, Corradi D, Sharma AM (2005) Epicardial adipose tissue: anatomic, biomolecular and clinical relationships with the heart. Nature Clinical Practice Cardiovascular Medicine 2(10): 536-543.

2. Corradi D, Maestri R, Callegari S, Pastori P, Goldoni M, et al. (2004) The ventricular epicardial fat is related to the myocardial mass in normal, ischemic and hypertrophic hearts. Cardiovascular Pathology 13(6): 313316 .

3. Iacobellis G, Malavazos AE, Corsi MM (2011) Epicardial fat: from the biomolecular aspects to the clinical practice. The International Journal of Biochemistry \& Cell Biology 43(12): 1651-1654.

4. Iacobellis G, Bianco AC (2011) Epicardial adipose tissue: emerging physiological, pathophysiological and clinical features. Trends in Endocrinology and Metabolism 22(11): 450-457.

5. Prati F, Arbustini E, Labellarte A, Sommariva L, Pawlowski T, et al. (2003) Eccentric atherosclerotic plaques with positive remodelling have a pericardial distribution: a permissive role of epicardial fat? A threedimensional intravascular ultrasound study of left anterior descending artery lesions. European Heart Journal 24(4): 329-336.

6. Sacks HS, Fain JN, Holman B, Cheema P, Chary A, et al. (2009) Uncoupling protein-1 and related messenger ribonucleic acids in human epicardial and other adipose tissues: epicardial fat functioning as brown fat. The Journal of Clinical Endocrinology \& Metabolism 94(9): 3611-3615.

7. Sacks HS, Fain JN, Bahouth SW, Ojha S, Frontini A, et al. (2013) Adult epicardial fat exhibits beige features. The Journal of Clinical Endocrinology \& Metabolism 98(9): 1448-1455.

8. Fitzgibbons TP, Czech MP (2014) Epicardial and perivascular adipose tissues and their influence on cardiovascular disease: basic mechanisms and clinical associations. Journal of the American Heart Association 3(2): e000582.

9. Marchington JM, Pond CM (1990) Site-specific properties of pericardial and epicardial adipose tissue: the effects of insulin and high-fat feeding on lipogenesis and the incorporation of fatty acids in vitro. International Journal of Obesity 14(12): 1013-1022.

10. Pezeshkian M, Noori M, NajjarpourJabbari H, Abolfathi A, Darabi M, et al. (2009) Fatty acid composition of epicardial and subcutaneous human adipose tissue. Metabolic Syndrome and Related Disorders 7(2): 125131.

11. Mazurek T, Zhang L, Zalewski A, Mannion JD, Diehl JT, et al. (2003) Human epicardial adipose tissue is a source of inflammatory mediators. Circulation 108(20): 2460-2466.

12. Baker AR, Silva NF, Quinn DW, Harte AL, Pagano D, et al. (2006) Human epicardial adipose tissue expresses a pathogenic profile of adipocytokines in patients with cardiovascular disease. Cardiovascular Diabetology 5: 1 .

13. Turer AT, Scherer PE (2012) Adiponectin: mechanistic insights and clinical implications. Diabetologia 55(9): 2319-2326.

14. Fang X, Palanivel R, Cresser J, Schram K, Ganguly R, et al. (2010) An APPL1-AMPK signaling axis mediates beneficial metabolic effects of adiponectin in the heart. American Journal of Physiology-Endocrinology and Metabolism 299(5): 721-729.

15. McAninch EA, Fonseca TL, Poggioli R, Panos AL, Salerno TA, et al. (2015) Epicardial adipose tissue has a unique transcriptome modified in severe coronary artery disease. Obesity (Silver Spring) 23(6): 1267-1278.

16. Iacobellis G, Ribaudo MC, Zappaterreno A, Iannucci CV, Leonetti F (2004) Relation between epicardial adipose tissue and left ventricular mass. American Journal of Cardiology 94(8): 1084-1087.

17. Malavazos AE, Di Leo G, Secchi F, Lupo EN, Dogliotti G, et al. (2010) Relation of echocardiographic epicardial fat thickness and myocardial fat. American Journal of Cardiology 105(12): 1831-1835.

18. Perticone F, Ceravolo R, Candigliota M, Ventura G, Iacopino S, et al. (2001) Obesity and body fat distribution induce endothelial dysfunction by oxidative stress: protective effect of vitamin C. Diabetes 50(1): 159165.

19. Steinberg HO, G Paradisi, G Hook, K Crowder, J Cronin, et al. (2000) Free fatty acid elevation impairs insulin-mediated vasodilation and nitric oxide production. Diabetes 49(7): 1231-1238. 
20. Abel ED, Doenst T (2011) Mitochondrial adaptations to physiological vs. pathological cardiac hypertrophy. Cardiovascular Research 90(2): 234242.

21. Belke DD, Larsen TS, Gibbs EM, Severson DL (2000) Altered metabolism causes cardiac dysfunction in perfused hearts from diabetic $(\mathrm{db} / \mathrm{db})$ mice. American Journal of Physiology-Endocrinology and Metabolism 279(5): 1104-1113.

22. Hirata Y, Tabata M, Kurobe H, Motoki T, Akaike M, et al. (2011) Coronary atherosclerosis is associated with macrophage polarization in epicardial adipose tissue. Journal of the American College of Cardiology 58(3) 248-255.

23. Salgado Somoza A, Teijeira Fernández E, Fernández AL, González Juanatey JR, Eiras S (2010) Proteomic analysis of epicardial and subcutaneous adipose tissue reveals differences in proteins involved in oxidative stress. American Journal of Physiology-Heart and Circulatory Physiology 299(1): 202-209.

24. Kajimura S, Spiegelman BM, Seale P (2015) Brown and Beige Fat: Physiological Roles beyond Heat Generation. Cell Metabolism 22(4): 546-559.

\section{ISSN: 2574-1241}

DOI: 10.26717/BJSTR.2019.12.002271

Ching Feng Cheng. Biomed J Sci \& Tech Res

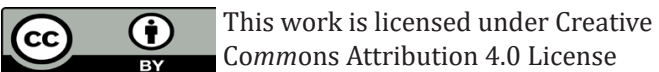

Submission Link: https://biomedres.us/submit-manuscript.php
25. Townsend K, Tseng YH (2012) Brown adipose tissue: Recent insights into development, metabolic function and therapeutic potential. Adipocyte 1(1): 13-24.

26. Berbee JF, Boon MR, Khedoe PP, Bartelt A, Schlein C, et al. (2015) Brown fat activation reduces hypercholesterolaemia and protects from atherosclerosis development. Nature Communications 6: 6356.

27. Dozio E, Vianello E, Briganti S, Fink B, Malavazos AE, et al. (2014) Increased reactive oxygen species production in epicardial adipose tissues from coronary artery disease patients is associated with brownto-white adipocyte trans-differentiation. International Journal of Cardiology 174(2): 413-414.

28. Gunawardana SC, Piston DW (2015) Insulin-independent reversal of type 1 diabetes in nonobese diabetic mice with brown adipose tissue transplant. American Journal of Physiology-Endocrinology and Metabolism 308(12): E1043-1055.

$\begin{array}{ll}\text { BIOMEDICAL } & \text { Assets of Publishing with us } \\ \text { RESEARCHES } & \text { - Global archiving of articles } \\ & \text { - Immediate, unrestricted online access } \\ & \text { - Rigorous Peer Review Process } \\ \end{array}$

